Meta

Journal des traducteurs

Translators' Journal

\title{
Dictionnaires multilingues généraux en langues africaines
}

\section{Antoine Lipou}

Volume 42, numéro 2, juin 1997

Lexicologie et terminologie II (1) et Traduction et post-colonialisme en Inde

Translation and Postcolonialism: India (2)

URI : https://id.erudit.org/iderudit/003511ar

DOI : https://doi.org/10.7202/003511ar

Aller au sommaire du numéro

Éditeur(s)

Les Presses de l'Université de Montréal

ISSN

0026-0452 (imprimé)

1492-1421 (numérique)

Découvrir la revue

Citer cet article

Lipou, A. (1997). Dictionnaires multilingues généraux en langues africaines. Meta, 42(2), 264-272. https://doi.org/10.7202/003511ar

\section{Résumé de l'article}

Le but de cet article est d'esquisser à grands traits les caractères généraux des traditionnels dictionnaires multilingues africo-européens. L'analyse prend appui sur les pratiques lexicographiques congolaises. S'il apparaît que l'instrumentation d'une langue s'assoit sur une connaissance scientifique de sa systématique, la même exigence s'impose en matière lexico-graphique ; l'état actuel des connaissances en sémantique, en lexicologie, en traduction et dans les autres sciences connexes le permet. 


\title{
DICTIONNAIRES MULTILINGUES GÉNÉRAUX EN LANGUES AFRICAINES*
}

ANTOINE LIPOU

Université Marien Ngouabi, Brazzaville, Congo

\begin{abstract}
Résumé
Le but de cet article est d' esquisser à grands traits les caractères généraux des traditionnels dictionnaires multilingues africo-européens. L'analyse prend appui sur les pratiques lexicographiques congolaises. S'il apparait que l'instrumentation d'une langue s'assoit sur une connaissance scientifique de sa systématique, la même exigence s' impose en matière lexicographique; l'état actuel des connaissances en sémantique, en lexicologie, en traduction et dans les autres sciences connexes le permet.
\end{abstract}

\begin{abstract}
This article presents a general overview of traditional African-European multilingual dictionaries with specific reference to lexicographic research techniques used in the Congo. If language description is based on a scientific knowledge, the same applies to lexicographic research. Current findings in semantics, lexicography, translation and the sciences can be helpful in this respect.
\end{abstract}

\section{INTRODUCTION}

Dans une problématique de maintenance des dictionnaires multilingues africanoeuropéens requise par les nécessités d'instrumentation des langues africaines, les perspectives d'amélioration de ces outils appellent un réexamen des problèmes d'ordre contrastif qui n'ont reçu que peu ou prou d'attention. L'une des questions soulevées porte sur la connaissance des limites de la couverture sémantique des articles; ce point acquiert une importance majeure et décisive eu égard au rôle, à la place et aux objectifs qui peuvent être assignés aujourd'hui aux traditionnels dictionnaires multilingues africano-européens, dans un contexte de nouveaux besoins de communication.

Le but de ce texte est d'esquisser à grands traits leurs caractères généraux : vision des faits lexicaux, fondements des approches lexicographiques et leurs limites. L'analyse prendra appui sur les pratiques lexicographiques congolaises. La justification de cette approche est à la fois théorique et pratique. S'il apparaît que l'instrumentation d'une langue s'assoit sur une connaissance scientifique de sa systématique (phonologique, morphologique et syntaxique), la même exigence s'impose en matière lexicographique; celle-ci implique un travail de description contrastive du sens des unités pour sortir le travail lexicographique d'un certain empirisme; l'état actuel des connaissances en sémantique, en lexicologie, en traduction et dans les autres sciences connexes le permet.

\section{CARACTÈRES GÉNÉRAUX}

La lexicographie congolaise, vieille de trois siècles, s'est constituée dans le sillage épistémologique de la Renaissance humaniste dont elle assume encore l'héritage. Les bouleversements de la lexicologie du $\mathrm{XX}^{\mathrm{e}}$ siècle n'ont fondamentalement rien modifié aux pratiques établies. Celles-ci sont demeurées longtemps l'apanage des missionnaires, lexicographes amateurs ou professionnels.

Meta, XLII, 2, 1997 
La tradition du dictionnaire multilingue remonte au XVII ${ }^{\mathrm{e}}$ siècle. C'est en 1651 qu'elle voit le jour avec la publication du Vocabulaire Kikongo-Latin-Espagnol de l'abbé Ghell. Ce document inaugure l'ère du dictionnaire de traduction qui va opérer le rapprochement des langues proches et lointaines, orales et écrites, communes et dialectales, vivantes et mortes, indo-européennes (latin, portugais, français, anglais, espagnol et suédois) et africaines (bantu et oubanguiennes).

Les termes «dictionnaire, lexique, glossaire, vocabulaire» sont utilisés indifféremment pour désigner des modalités d'enregistrement ou de fixation écrite d'une matière lexicale provenant tout aussi bien de l'usage courant que des vocabulaires spécialisés. Ces instruments apparaissent comme nécessairement normatifs du double point de vue du choix idéologico-moral des données constitutives de leur macro-structure et des principes de leur organisation en un ordre alphabétique immuable.

La dimension du multilinguisme prise en compte varie d'un document à l'autre. La mise en rapport peut concerner plus de quatre langues ou variétés d'un même continuum géographique, notamment quand le dictionnaire est à la fois multilingue et multidialectal. La grille classique qui aborde les questions de traduction en termes de thème (traduction dans la langue non-maternelle) et de version (traduction dans la langue maternelle), s'avère inadéquate à rendre compte de la diversité des combinaisons lexicographiques apparentes dans le corpus. Au regard de la dynamique sociolinguistique observable sur le terrain, la dichotomie langue maternelle/langue non-maternelle est transcendée par la complexité de la réalité de la langue première : si elle est généralement la langue maternelle des géniteurs ou celle de l'un d'eux, elle peut être une langue véhiculaire régionale, ou encore la langue française pour certains sujets. Le développement des compétences multiples en langue seconde, troisième, voire quatrième est un autre aspect de la question.

En somme, la vision du «dictionnaire» qui s'est ainsi imposée a privilégié l'ouvrage d'aide à la traduction; ce dernier est à la fois multilingue, contrastif, général, spécialisé, synchronique, alphabétique, normatif, et à équivalences statiques.

Les synthèses bibliographiques disponibles (Alac-Congo 1987) laissent apparaître quatre types d'outils lexicographiques qui peuvent être qualifiés de dictionnaires de premières traductions, de retraductions et d'hétéro-traductions.

\section{TYPOLOGIE DES DICTIONNAIRES MULTILINGUES}

\section{Le type strictement bilingue}

Il met en parallèle une langue congolaise (comme source) et une langue indoeuropéenne (comme cible); et vice-versa: Bonneau (1956); Calloc'h (1911a). Cette approche donne lieu à la confection soit de deux documents distincts soit d'un ouvrage unique qui combine le double rapport de traduction allant d'une langue congolaise vers une langue indo-européenne et d'une langue indo-européenne vers une langue congolaise : Butaye (1909), Craven (1883).

\section{Le type trilingue}

C'est sous cette forme que fut conçu et diffusé en 1651 le plus ancien outil lexicographique connu, non seulement d'une langue congolaise mais aussi d'une langue bantu, le Vocabulaire kikongo-latin-espagnol de l'abbé P. S. Ghell, mentionné plus haut. Le type trilingue présente également différentes variantes. Les combinaisons observées associent deux langues indo-européennes et une langue congolaise (Diener et Maillard 1970), une langue congolaise et deux langues indo-européennes (Fehderau 1969), ou encore une langue indo-européenne et deux langues africaines dont l'une n'est pas d'usage au Congo (Maia 1964). 


\section{Le type quadrilingue}

L'organisation de la relation de traduction semble avoir privilégié l'optique de la langue indo-européenne vers les langues africaines. Ces dernières peuvent appartenir à un même ensemble historique et structural (Calloc'h 1911b); elles peuvent ne pas avoir de parenté immédiate (Giraud 1900).

\section{Le type multilingue et multidialectal}

Il apparaît dans les travaux qui consignent dans un même document des données fournies par tout un ensemble de parlers ayant des affinités structurales, géographiques et historiques. C'est particulièrement au domaine des parlers bantu H.10. (groupe kongo) que cette approche a été appliquée (Laman 1936). Cette démarche est marquée par l'influence du comparatisme génétique du XIX ${ }^{e}$ siècle, soucieux de la quête de la «langue uniforme» et de la «langue de référence» qui émergeraient de la réduction des variations géographiques du continuum. Laman a été lui-même disciple du néo-grammairien et bantuiste C. Meinhof. Pour sa part, Swartenbroeckx (1973) a ajouté à l'aspect multidialectal la dimension véhiculaire, c'est-à-dire l'hétérogénéité agissant dans le groupe linguistique étudié. Le fait véhiculaire, façonné par le contexte de multilinguisme, est porteur d'une forte diversification des sources lexicales; il développe la synonymie hybride. Sous cet angle, il brise la cohérence de la grille d'analyse qui présuppose l'homogénéité du continuum linguistique, mis en corrélation avec un territoire, un groupe humain et un passé historique.

\section{CONTENU DE LA MACRO-STRUCTURE}

L'organisation de la nomenclature se conforme invariablement à l'ordre alphabétique roman. Lorsque la langue source est une langue africaine, les faits traités qui s'accommodent mal de cette organisation soulèvent deux difficultés majeures.

La première touche aux rapports disjonctifs de la graphie et de la phonie: des lettres de l'alphabet du genre C, H, Q, R, X ont été utilisées comme têtes de chapitre; sous elles ont été enregistrés des emprunts ou quelques commentaires. La conséquence immédiate, c'est l'apparition de l'hybridité graphique qui fait voisiner l'orthographe française (par exemple) avec la transcription phonétique. Fehderau (1969) a consigné sous la lettre C des emprunts français ayant ce symbole comme initiale graphique. Dans le même temps, les variantes de ces emprunts, en tant que formes intégrées dans la langue réceptrice, sont retranscrites phonétiquement. C'est ainsi que cohabitent les doublets de type «camarade» et «kamaladi», «commencer» et «komase», «chaussette» et «soseti», ou encore «lunette» et «luneti». La transcription phonétique des emprunts réaménagés en langue réceptrice se plie au principe de correspondance d'un son à un graphème; les vocables qui ont conservé l'orthographe française obéissent à des règles d'un autre ordre: "commencer» comporte deux «m», à l'inverse de «komase». Cette hétérographie est source de confusion dans l'interprétation de certains symboles qui peuvent être considérés soit comme des isographèmes, soit comme graphes uniques à lecture variable. Le graphème $u$, repris dans «lunette» et dans «luneti», reçoit à la fois la valeur de voyelle palatale antérieure arrondie du système vocalique français $[\mathrm{y}]$ et celle de voyelle postérieure arrondie du vocalisme du munukutuba [u].

La deuxième observation que suscite l'ordre alphabétique a trait au dégroupement des champs lexicaux et dérivationnels. Les lexèmes sont souvent repris sous des entrées différentes qui correspondent aux initiales de leurs préfixes nominaux. Prenons en exemple l'équivalent du terme français «deuil». 11 correspond au lexème dilu et apparaît chez Fehderau (1969) sous la lettre B à l'entrée bidilu et sous la lettre K à la vedette kidilu. Les préfixes nominaux bi- et ki- sont en opposition de nombre. À l'inverse, les lexèmes à 
préfixe unique du genre -lendo dans lulendo (orgueil, arrogance), -mputu dans bumputu (pauvreté) ou -bela dans kubela (tomber malade) ne connaissent qu'un seul enregistrement.

La première conséquence de cette vision alphabétique des données lexicales est donc la multiplication du nombre d'entrées pour un même lexème. La seconde, c'est l'éclatement des champs dérivationnels; il concerne les lexèmes qui, par un jeu formel et sémantique de substitution de leurs préfixes, construisent des micro-systèmes. Les éléments formés à l'aide du lexème -long- (notion d'apprendre ou d'enseigner) sont distribués sous les lettres alphabétiques $\mathrm{K}, \mathrm{M}, \mathrm{N}$. Le graphème $\mathrm{K}$ enregistre comme entrées distinctes kulonga (apprendre), kulongisa (enseigner), kulongama (être éduqué)... La lettre $\mathrm{M}$ comporte malongi (conseils), mulongi (pédagogue) milongi (pédagogues), mulonga (rang)... La lettre $\mathrm{N}$ atteste les vedettes $n$ dongokolo (apprentissage), ndongosolo (manière d'enseigner).

\section{MICRO-STRUCTURE}

Le contenu de l'article demeure fort variable d'un document à un autre. Il existe deux types d'articles. L'un se réduit à un simple relevé lexical qui se borne à mettre en parallèle les signes linguistiques des langues rapprochées. L'autre, un peu plus élaboré, s'efforce d'offrir des informations de divers ordres.

a) Quelques dictionnaires illustrent l'emploi de la vedette à l'aide de séquences d'énoncés. Parmi les très rares documents qui adoptent cette démarche figure le Dictionnaire kituba-français de Kusopo et Tollenier (1973).

b) Les collocations et les idiomatismes sont quelquefois partie intégrante de l'article. Si l'approche n'a pas été systématique dans son Dictionnaire kikongo ya leta, Fehderau (1969) propose un très large éventail d'illustrations du fonctionnement des champs sémantiques de certaines unités lexicales. C'est ainsi que sous l'entrée pesa (donner) ont été reproduites plus de 30 collocations dont on peut extraire pesa inswa (donner la permission), pesa kidimbu (donner quelque chose en caution), pesa kiese (rendre heureux), pesa kivumu (féconder), pesa lufutu (rémunérer), pesa lusakumunu (donner une bénédiction), pesa luzitu (respecter), pesa maboko (donner un coup de main), pesa malongi (donner des conseils), pesa matondo (remercier), pesa mvutu (donner une réponse)...

c) Quelques faits morphologiques apparaissent également. Il s'agit essentiellement de données de morphologie dérivationnelle qui ont trait à l'appariement des préfixes nominaux et aux extensions; l'entrée mubuki (moissonneur) est immédiatement suivie du préfixe ba- qui commande la formation de l'élément babuki (moissonneurs), à valeur de pluriel.

d) Les indications relatives à la distribution géographique des variations sémantiques de la vedette sont mentionnées dans les dictionnaires de traduction multilingues et multidialectaux. Généralement, ces documents présentent en début d'ouvrage la carte géographique du continuum étudié. Dans le corps de l'article, les variations sémantiques sont affectées d'un des symboles des quatre points cardinaux, spécifiant ainsi les écarts géographiques dans les usages.

e) L'origine linguistique des emprunts est signalée dans bon nombre de documents. Cette information est ordinairement donnée à propos des langues indo-européennes; elle l'est éventuellement dans les situations où il est apparu aisé d'établir la source d'un emprunt à une autre langue africaine.

f) L'explication et le discours métalinguistique occupent une place de choix dans certains cas. L'explication peut prendre la forme de la paraphrase : l'entrée bibila chez Fehderau (1969) a pour contenu les équivalents Bible [baibl] et Bible [bibl] en langues cibles, anglaise et française; la traduction est complétée par l'énumération des variantes formelles de l'entrée (mabibila, biblia, bible) et par la présence d'une paraphrase qui a valeur d'éclaircissement: mukanda ya nzambi (littéralement, la lettre de Dieu). Le commen- 
taire métalinguistique tient lieu de contenu de l'article quand l'entrée est beaucoup moins une unité lexicale qu'un morphème grammatical (affixe); 1'article troque son contenu traductionnel contre une glose. Chez Fehderau (1969: 141), les entrées m- et ma- ont été présentées de la manière suivante: sous $\mathrm{m}-$, on lit «Préfixe singulier nominal; le pluriel est ba-»; les différentes entrées ma- sont commentées tour à tour comme «Préfixe nominal pluriel qui détermine la classe ma- des substantifs» et comme «Préfixe de dérivation qui forme un substantif d'un autre substantif indiquant une occurrence de cette qualité. Il s'emploie dans la région orientale. Il forme un substantif invariable.»

Ces types d'entrées soulèvent un double problème; celui du statut linguistique des données qu'enregistre le dictionnaire et celui de son ouverture vers des grammaires lexicales.

g) La mention des champs conceptuels de l'entrée complète parfois l'équivalence. Commandeur (1968: 118-119) organise sous la vedette «poisson» toute la taxinomie des poissons, tels qu'ils sont distingués dans la langue cible. La même approche a été appliquée au traitement d'autres entrées comme «banane, bois, mère, manioc, maladie, noix». Ces illustrations confèrent au document une certaine dimension de dictionnaire encyclopédique.

\section{FONDEMENTS ÉPISTÉMOLOGIQUES DE LA TRADUCTION}

Cette lexicographie comparative n'a jamais énoncé de manière explicite les fondements de la mise en parallèle des langues qu'elle rapproche. La recherche des équivalences ou des correspondances prend appui sur la compétence linguistique multiple, réelle ou supposée du lexicographe lui-même, ou sur celle de ses informateurs ; le recours à la compilation des documents préexistants demeure également l'une des sources utilisées. Examiner les modes d'établissement des équivalences, c'est s'interroger sur les présupposés qui leur confèrent leur fondement épistémologique et heuristique. Leur connaissance éclaire davantage la saisie des motivations qui président à la sélection des équivalences.

Ces dernières sont recherchées au niveau des signes et des signifiés des langues mises en parallèle, en prenant comme principes l'isolement de l'unité lexicographique, son univocité, la corrélation réciproque des unités lexicales des langues comparées, et l'isomorphisme des contenus référentiels.

a) La décontextualisation de l'unité lexicographique est un fait classique de cette pratique lexico-traductionnelle qui recherche des équivalences statiques, hors de tout cadre discursif ou énonciatif. Il existerait ainsi des signifiés stabilisés. Ce serait une condition nécessaire à l'établissement de l'équivalence comme reconstruction de la «signification» à un point de convergence, supposé, des unités lexicales de la langue source et de la langue cible. Cela soulève naturellement le problème de la validité du concept d' «équivalence de traduction», lorsque celle-ci est perçue comme relevant des phénomènes éminemment pragmatiques (Roberts et Pergnier 1987).

b) La décontextualisation et la fixité présumées de son contenu sémantique ne confèrent à l'unité lexicale que la capacité d'être monosémique. Ce contenu est censé lui correspondre, conformément à une ancienne conception du lexique qui en faisait une simple nomenclature. Cette démarche procède par élagage des valeurs sémantiques attachées à chaque signe pour n'en privilégier qu'une seule ou éventuellement quelques-unes, celle(s) sur laquelle ou sur lesquelles s'appuie la relation de correspondance hétéroglossique. L'existence de l'unité lexicographique n'est pensée possible que grâce à cette supposée univocité.

c) La corrélation réciproque découle de cette prétendue monosémie invariable et absolue qui serait communément partagée par les langues comparées. La relation qui est posée entre l'entrée et son ou ses équivalents se saisirait comme fondée sur une identité de champ sémantique. 
d) L'équivalence est envisagée sur la base du pressentiment de l'existence d'un découpage référentiel isomorphe. Le principe de monoréférentialité s'ajoute ainsi aux autres éléments constitutifs de la base heuristique de reconstruction de la signification en langue cible.

e) Le principe d'existence d'une «nature universelle» énonce que par delà les différences d'enracinement écologique ou culturel, la «nature universelle» de certains concepts et de certaines expériences rend aisée la recherche des équivalences.

\section{RELATIONS DE TRADUCTION}

La description contrastive des contenus sémantiques qui est entreprise ici s'inscrit dans une perspective sémantico-référentielle; elle se limite à l'aspect dénotatif de la relation de sens, révélée par l'échantillon de dictionnaires soumis à l'analyse. Dans cette optique, il est question de comparer les informations foumies par les unités lexicales en langues source et cible(s) à propos d'un même objet, d'un même événement, d'un même état des choses. Ces informations seront interprétées en termes de propriétés sémantiques dénotatives, modélisées dans chacune des lexies examinées.

Trois cas de figure président à l'organisation des relations de traduction. Le premier laisserait présager que l'entrée et son correspondant partageraient une équivalence bâtie sur les mêmes propriétés dénotatives.

Le second est caractérisé par un partage partiel des propriétés lexicalisées par chacun des vocables mis en correspondance.

Le troisième manifeste une non-identité d'informations entre l'entrée et son ou ses «équivalent(s)». Ces trois approches différentes et concurrentes coexistent.

\section{Le premier cas}

Héritière de la Renaissance humaniste, la lexicographie congolaise s'est enfermée dans ce que Rastier (1991 : 38) nomme «une conception traductionniste du sens». Celle-ci retient du «sens d'un symbole [...] sa traduction en d'autres symboles». Tout cela débouche sur une imbrication complexe des rapports de traduction qui s'établissent entre les représentations du référent et les signes linguistiques correspondants dans les langues rapprochées, entre les signes linguistiques de la langue de départ et ceux qui les représentent dans la langue d'arrivée.

Inférer une relation d'équivalence entre ces deux types de signes conduirait à penser qu'il y a identité d'informations à différents paliers de toute cette «superposition de représentations symboliques». On rechercherait ainsi les conditions de «traduisibilité et de traductivité» dans l'identité des paramètres référentiels (certainement le plus immédiatement accessible), sémantique, stylistique, connotatif... La possibilité d'une identité d'information requérait que l'opération de traduction intègre systématiquement le plus grand (si ce n'est le même) nombre de traits notionnels, sémantiques, stylistiques... véhiculés par les signes de la langue source.

Un tel traitement lexicographique procède beaucoup plus par perception globale de la conceptualisation du référent dans la langue source.

Il prend appui sur la présupposition de l'isomorphisme de contenu monoréférentiel entre les éléments rapprochés. La relation de traduction qui en découle s'exprime par une correspondance de terme à terme; celle-ci se rencontre dans les domaines comme ceux des phénomènes météorologiques (arc-en-ciel, foudre, tonnerre, éclair), des sécrétions corporelles (salive, urine, larme), des minéraux (charbon, fer, pierre), de la géographie (colline, forêt, rive). Mais il s'opère également des transferts ou des projections d'un grand nombre ou de la totalité des traits notionnels de l'unité de départ sur celle de la langue d'arrivée. Cela est apparent dans les exemples où deux vocables qui sont tenus distincts dans la 
langue source reçoivent le même correspondant dans la langue cible. Commandeur (1968) propose pour les termes français «brouillard» et «brume» le même équivalent «dibungi» ou «mbugi»; les deux vocables de départ sont supposés lexicaliser le même contenu référentiel auquel renvoie leur équivalent traductionnel commun.

\section{Le deuxième cas}

Il s'appréhende comme une situation de proximité ou de partage partiel des propriétés dénotatives véhiculées par les vocables des langues mises en parallèle. La proximité de contenu sémantico-référentiel peut être fondée sur une perception sélective du concept de départ. L'opération de traduction ne retient que certains ou quelques traits de l'élément de départ. Le traitement du champ lexical de coiffure chez Commandeur (1968) peut servir d'illustration. Les entrées «bonnet», «casque», «casquette», «chapeau», «chéchia» reçoivent le même équivalent traductionnel qui est «impu». Ceci appelle quelques commentaires. Tout d'abord, la stratégie lexicographique ne retient de l'information fournie en langue de départ que le trait générique de coiffure comme artefact servant à couvrir la tête ou à l'orner. C'est par le biais de cette propriété sémantico-conceptuelle qu'est perçue puis établie en langue cible la synonymie référentielle des vocables français. Ensuite, le contenu dénotatif rattaché à cette synonymie dicte à son tour le choix de l'équivalent unique. Enfin, il apparaît qu'il y a eu en langue cible une déperdition d'informations tant du point de vue de certains contenus connotatifs (les sens dits figurés) que de celui des valeurs sémantiques différenciatrices de départ. Les traits notionnels, distinctifs ou spécifiques, de chacun des termes du champ lexical de coiffure ont été neutralisés dans l'opération de traduction. Il s'agit des propriétés qui sont liées aux fonctions de la coiffure (protection pour le casque, ornement pour la chéchia, distinction pour la casquette), à la matière de fabrication (tissu pour le bonnet, métal ou cuir bouilli pour le casque), à la forme (rigidité pour le casque, souplesse pour le bonnet, hauteur pour le chapeau, cylindricité pour la chéchia, rotondité). La mise en parenthèses de ces traits débouche sur l'évacuation d'autres domaines d'emploi dont relèvent certains termes: le vocable «casque» possède un autre trait, celui de «dispositif qui coiffe la tête». Ce trait qui s'applique au domaine touchant aux casques (téléphonique, à écouteurs, de radio, ou à air chaud pour sécher les cheveux) n'a pas été pris en compte dans l'opération de traduction.

Le champ lexical des termes de coiffure se présente ainsi comme l'un des multiples exemples d'identité partielle des dimensions descriptives du référent qu'offrent la langue source et la langue cible. La multiréférentialité qui est conférée à l'équivalent unique en fait une unité lexicale polysémique.

\section{Le troisième cas}

Il regroupe différents types de décalages constatés entre les unités lexicales des langues sources et celles des langues cibles. Le décalage provient des différences dans la modélisation du référent. Trois situations de décalage se présentent, selon qu'en langue cible il est offert davantage, moins, ou d'autres informations dénotatives.

- La première trouve une abondante illustration dans les zones lexicales des termes des relations sociales ou des représentations collectives, lorsque les langues cibles sont langues congolaises. Commandeur (1968) propose pour le terme français «président» l'équivalent «mfumu». Le champ sémantique et référentiel de mfumu a un spectre beaucoup plus large. Il couvre les notions d'exercice du pouvoir temporel et spirituel, de statut de personne libre (par opposition à celui d'esclave), de puissance économique, d'influence ou de poids moral qu'un individu peut avoir dans sa société... Ce terme qualifie ainsi un être riche, puissant, libre, jouissant d'une certaine autorité mais aussi déifié. Mettre en parallèle «président» et «mfumu», c'est rapprocher des vocables qui sont structurés différemment. S'il est vrai que les deux termes ont en commun les traits notionnels «puissance, 
autorité, obligation d'allégeance faite aux gouvernés», le personnage «président de la République» évoque d'autres propriétés qui lui sont rattachées: les procédures électives qui conditionnent l'accès au pouvoir, les prérogatives constitutionnelles reconnues, le cadre statutaire d'exercice des fonctions de chef d'État... Ces propriétés qui tiennent de l'État de type occidental ne sont pas configurées dans l'équivalent «mfumu».

- La seconde situation de décalage est attestée dans plusieurs documents recensés. Dans son dictionnaire d'hétéro-traductions, Fehderau (1969) retient comme équivalents de l'entrée «lúto», les expressions anglaise «skin disease (round, red patches)» et française «maladie de peau (plaques rondes et rougeâtres)». En termes taxinomiques, «lúto» est un vocable spécifique. Les traits référentiels qu'il structure mettent en évidence les manifestations de cette dermatose (démangeaison, plaques, desquamation), les zones du corps qui sont atteintes (visage, cage thoracique, dos) et ses causes si l'on entre dans le domaine de l'anthropologie de la maladie. Les deux traductions «skin disease» et «maladie de peau» qui sont convergentes s'en tiennent à des traits notionnels très généraux que tentent de compléter les explications données entre parenthèses. Il reste que les formules paraphrastiques utilisées dans les langues cibles auraient pu être remplacées par des termes généraux comme pityriasis ou dartre.

- La dernière situation met en évidence des exemples de traduction dans lesquels les informations dénotatives à l'arrivée ne rendent pas compte du contenu qui préexiste au départ. Il est des cas où la difficulté provient de la distance culturelle; par conséquent, le lexicographe est confronté aux différences de structuration sémantico-référentielle entre les langues comparées. D'autres cas tiennent des données insuffisamment documentées. Commandeur (1968) traduit le terme français «séducteur» par «muyibi». Si l'on s'en tient aux deux acceptions que donne Le Petit Robert, le mot «séducteur» est synonyme de «corrupteur» et de «homme à femmes, lovelace, tombeur». Le vocable «muyibi» qui est retenu en langue cible a le sens de «voleur ou voleuse».

Il existe dans le même document d'autres exemples d'équivalence qui illustrent la situation extrême du décalage. La vedette «mahométan» a été traduite par «haussa». Ce choix résulte du télescopage d'un phénomène religieux et d'un fait linguistique. Par «mahométan», est désignée toute personne qui professe la religion de Mahomet ou y adhère. Le terme «haussa» renvoie à une réalité linguistique: le hausa est une langue d'Afrique de l'Ouest jouissant du statut de lingua franca. La motivation de l'équivalence établie entre «mahométan» et «haussa» réside dans la perception populaire qui a cours au Congo et qui assimile tout commerçant venu d'Afrique de l'Ouest à un (locuteur) haussa.

\section{CONCLUSION}

Ce texte s'est attaché à présenter un tableau panoramique des limites les plus apparentes des outils lexicographiques en langues congolaises. Les causes en sont multiples; elles relèvent tout autant du poids de certaines traditions heuristiques que de l'état des connaissances théoriques des lexicographes praticiens.

Le rôle essentiel que sont appelés à jouer les dictionnaires multilingues (comme les monolingues) n'est plus à démontrer. Outre les domaines traditionnels de l'évangélisation, de nouvelles sphères de communication institutionnelle requièrent l'utilisation d'outils lexicographiques mieux élaborés et plus opérationnels. Les médias - notamment la presse parlée dans les langues nationales - sont un secteur où la demande en outils d'aide à la traduction s'avère très forte, au regard du traitement de l'actualité quotidienne ou des programmes spéciaux de la radio éducative (agriculture, alphabétisation fonctionnelle...). Ces besoins dictent de nouvelles orientations en matière de confection de dictionnaires ou de répertoires multilingues. La nécessité d'introduire des versions repensées s'impose; celles-ci seraient conçues selon la triple exigence de conformité à l'état actuel des connaissances 
scientifiques, à la spécificité du secteur de communication en cause, et aux structures des langues. L'exigence scientifique conduit à considérer que la dose de subjectivité toujours présente dans l'appréciation du degré d'équivalence n'exclut pas la possibilité d'une description systématique, cohérente et techniquement acceptable de la structuration des aires sémantiques lexicographiquement comparées. Cette description préalable des lexies rapprochées paraît être une condition nécessaire à la recherche de l'identité sémantique et de l'équivalence; par ailleurs, les déterminations historiques et culturelles qui interfèrent sur l'horizon de restitution des aires référentielles mériteraient d'être prises en considération.

\section{Note}

* Cet article est issu d'une communication présentée par l'auteur aux IVes Journées scientifiques du réseau «Lexicologie, terminologie, traduction» de l'AUPELF-UREF (Lyon, France, 28, 29, 30 septembre 1995).

\section{RÉFÉRENCES}

BONNEAU, J. (1956) : Grammaire pounoue et lexique pounou-français, Brazzaville, I.E.C., n $8,117 \mathrm{p}$. BUTAYE, R. (1909) : Dictionnaire kikongo-français, français-kikongo, Roulers, 800 p.

CALLOC'H, G. J. (1911a) : Vocabulaire français-ifumu (Batéké), précédé d'éléments de grammaire, Paris, Geuthner, $346 \mathrm{p}$.

CALLOC'H, G. J. (1911b) : Vocabulaire français-gmbwaga-gbanziri-monjombo, Paris, Geuthner, 204 p.

CRAVEN, D. (1883) : English-Congo and Congo-English Dictionary, London.

DIENER et MAILLARD (1970) : Petit vocabulaire français-anglais-munukutuba, Pointe-Noire.

FEHDERAU, H. W. (1969) : Dictionnaire kikongo (ya leta) anglais-français, Kinshasa, Leco, 323 p

GUIRAUD, G. (1900): Vocabulaires des dialectes Sango, Bakongo et A'zande, Paris, Challamel, 58 p.

HUOT, J.-Cl. (1980) : La distance interlinguistique lexicale, Québec, Publications du CIRB, Université Laval, $144+$ xi p.

KALONJI, T. Z. (1993) : La lexicographie bilingue en Afrique francophone : l'exemple français-ciluba, Paris, L'Harmattan, $367 \mathrm{p}$

KUSOPO, A. et P. TOLLENIER (1973) : Dictionnaire kituba-français, Kinshasa, Éditions Saint-Paul.

LAMAN, K. E. (1936): Dictionnaire kikongo-français, avec une étude phonétique décrivant les dialectes les plus importants de la langue dite kikongo, Bruxelles, 2 vol., $1183 \mathrm{p}$.

MAIA, P. A. S. (1964): Dictionario complementar portugues-kimbundu-kikongo, Lucuyaes, Missoes, 658 p.

MEYER, I. (1988): «The General Bilingual Dictionary as a Working Tool in Theme», Meta, 33 (3), septembre 1988 , pp. 368-376.

NIDA, E. A. (1975): Componential Analysis of Meaning and Introduction to Semantic Structure, The Hague, Mouton, $272 \mathrm{p}$.

RASTIER, F. (1991): Sémantique et recherches cognitives, Paris, PUF, 262 p.

ROBERTS, R. P. et M. PERGNIER (1987): «L'équivalence en traduction», Meta, 32 (4), décembre 1987, pp. 392-402.

SWARTENBROECKX, P. (1973) : Dictionnaire kikongo-kituba-français, Bandundu, Ceeba, série III, vol. 2, 815 p.

THOIRON, Ph. (1994) : «La terminologie multilingue : une aide à la maîtrise des concepts», Meta, 39 (4), décembre 1994, pp. 765-773.

TOPULOS, A. (1987) : «Introduction de la notion topologique pour la description du système notionnel» Meta, 32 (4), décembre 1987, pp. 442-448.

VAN HOOF, H. (1994) : «Les dictionnaires polyglottes - Esquisse historique», Meta, 39 (1), mars 1994, pp. 213-228. 